\title{
AVALIAC̃̃̃O DA RESPOSTA HEMATOLÓGICA DOS ANIMAIS TRATADOS COM Typha domingensis Pers E Operculina hamiltonii SOBRE NEMATÓIDES GASTRINTESTINAIS DE CAPRINOS
}

\author{
Evaluation of the hematological response of animals treated with Typha domingensis Pers and \\ Operculina hamiltonii on gastrointestinal nematodes of goats
}

\author{
Carpejane Ferreira da Silva ${ }^{1}$, Katiuscia Menezes da Silva Lôbo ${ }^{2}$, Ana Célia Rodrigues Athayde ${ }^{2}$, \\ Wilson Wolflan da Silva ${ }^{2}$, Edinaldo Queiroga de Lima², Nebson Fernandes Pequeno ${ }^{3}$
}

\begin{abstract}
RESUMO
Este estudo foi desenvolvido para avaliar a resposta hematológica dos animais submetidos aos tratamentos com Typha domingensis Pers (taboa) e Operculina hamiltonii (batata-de-purga), in natura, no controle das helmintoses gastrintestinais de caprinos naturalmente infectados. Foram utilizados 30 caprinos da raça moxotó. O rizoma da taboa e a raiz batata-de-purga foram usados para realização da fitoquímica. Os animais foram distribuídos em cinco tratamentos, com o grupo I tratados com moxidectina $0,2 \%$ em dose única de $0,5 \mathrm{mg} / \mathrm{Kg}$; grupo II com taboa na dose $10 \mathrm{~g} / 20 \mathrm{Kg}$ peso vivo; grupo III com batata-de-purga na dose de (09 $\mathrm{g} / 20 \mathrm{Kg}$ ); grupo IV com taboa $(10 \mathrm{~g} / 20 \mathrm{Kg}$ ) associada a batata-de-purga $(09 \mathrm{~g} / 20 \mathrm{Kg})$, durante três dias consecutivos e o grupo V foi o grupo controle. As fezes foram coletadas nos dias zero, sete e 25 para realização dos OPGs e para os exames hematológicos foram coletados sangue aos sete, 14 e 28 dias pós-tratamento, sendo avaliados hematócrito, hemoglobina, VGM e CHGM. Os valores do VGM, eritrócitos, hematócrito e hemogoblina ficaram dentro da normalidade para caprinos. Os tratamentos não afetaram os valores de VGM e CHGM. Quanto aos eritrócitos, hematócrito e hemogoblina houve interação entre período de coletas. Com a metodologia utilizada, o fornecimento de $T$. domingensis e $O$. hamiltonii não interferiu na resposta hematológica dos animais tratados.
\end{abstract}

Termos para indexação: Fitoterápicos, helmintos, hematologia.

\section{ABSTRACT}

This study evaluated the hematological response of animals submitted to treatments with Typha domingensis Pers and Operculina hamiltonii, in natura, in the control of gastrointestinal helminthiasis in goats naturally infected. Thirty moxotó goats were used. The rhizome and root of $T$. domingensis and $O$. hamiltonii, were used in the phytochemistry analysis. The animals were divided into five treatments with the group I treated with moxidectin $0.2 \%$ in a single dose of $0.5 \mathrm{mg} / \mathrm{kg}$, group II with $T$. domingensis using $10 \mathrm{~g} / 20 \mathrm{Kg}$ body weight, group III $O$. hamiltonii with a dosage of $09 \mathrm{~g} / 20 \mathrm{Kg}$, group IV with $T$. domingensis $(10 \mathrm{~g} / 20 \mathrm{Kg})$ associated with $O$. hamiltonii $(09 \mathrm{~g} / 20 \mathrm{Kg})$ during three consecutive days and the group $\mathrm{V}$ as the control. Feces were collected on days zero, seven and 25 for the FECs and for the hematological tests blood was collected at seven, 14 and 28 days post-treatment, for the evaluation of hematocrit, hemoglobin, MGV and MGHC. The values of MGV, erythrocytes, hematocrit and hemogoblin were within the normality for goats. The treatments had no effect on MGV and MGHC. Regarding the erythrocytes, hematocrit and hemoglobin, there was an interaction among periods of sampling. Using this methodology, the supply of $T$. domingensis and O. hamiltonii had no affect on the hematology response of treated animals.

Index terms: Fitoterapics, helminths, hematology.

(Recebido em 3 de fevereiro de 2010 e aprovado em 11 de novembro de 2010)

\section{INTRODUÇÃO}

Atualmente, o rebanho nacional e qualquer outro sistema de produção são acometidos por verminoses, sendo um dos problemas mais importantes na criação de ruminantes e responsáveis por grandes prejuízos na pecuária brasileira.

O frequente surgimento de surtos de verminoses na criação de caprinos no semiárido paraibano é o reflexo do modelo inadequado de controle utilizado pelos produtores. Erros do momento e na administração das dosagens, a persistência da utilização de antihelmínticos com o mesmo princípio químico e a falta de medidas adequadas de manejo, ocorrem por deficiência de informações técnicas adequadas que estejam disponíveis aos pecuaristas, contribuindo para um rápido desenvolvimento da resistência antihelmíntica.

\footnotetext{
1Universidade Federal de Campina Grande/UFCG - Centro de Saúde e Tecnologia Rural - Rodovia Patos Teixeira - s/n - Cx.P. 64 - 58700-970 Patos, PB - carpejanevet@hotmail.com

2Universidade Federal de Campina Grande/UFCG - Centro de Saúde e Tecnologia Rural - Patos, PB

3Universidade Federal Rural do Semi-Árido/UFERSA - Catolé do Rocha, PB
} 
Com isso, produtos e subprodutos derivados da agricultura orgânica, vêm se destacando na agropecuária, uma vez que, o uso de fitoterápicos origina produtos com menos resíduos e mais valorizados no mercado. A aplicação de substâncias vegetais pode causar um desenvolvimento mais lento da resistência, acreditando-se atingir somente espécies alvo, serem biodegradáveis e não causarem a poluição ambiental. Entretanto, estudos demonstram que metabólitos secundários de plantas (MSP) podem ter propriedades antiparasitárias, onde se tem evidenciado que taninos condensados, alcalóides e glicosídeos possuem a capacidade de exercer essas propriedades, no entanto, não se traduzem necessariamente em consequentes benefícios para ruminantes parasitados.

A razão é que a maioria dos MSP também tem propriedades antinutricionais e tóxicas, causando alterações fisiológicas temporárias ou permanentes em herbívoros (Harborne, 1999), o consumo excessivo pode induzir efeitos negativos relacionados às estas características (Athanasiadou \& Kyriazakis, 2004). Como tem sido demonstrado que taninos condensados reduzem a ingestão de alimento, a digestibilidade e o metabolismo do rúmen (Min et al., 2003), como também toxicidade das mucosas, redução na absorção de nutrientes, perda de peso e morte dos animais (Dawson et al., 1999; Waghorn \& McNabb, 2003).

O efeito tóxico desses metabólitos pode afetar importantes órgãos como o fígado, que é a fonte primária de vários componentes sorológicos incluindo uréia, albumina, glicose e a maioria dos fatores de coagulação (Val-Bicalho \& Carneiro, 2008).

Conduziu-se este trabalho, com o objetivo de avaliar a resposta hematológica dos animais submetidos ao tratamento com Typha domingensis Pers (taboa) e Operculina hamiltonii (G. Don) D.F. Austin \& Staples (batata-de-purga), in natura, no controle das helmintoses gastrintestinais de caprinos, naturalmente infectados em clima semiárido.

\section{MATERIAL E MÉTODOS}

O experimento foi realizado no Núcleo de Pesquisas para o Semiárido (NUPEÁRIDO) e nos laboratórios de Ciências Química e Biológicas (LCQB), Doenças Parasitárias dos Animais Domésticos (LDPAD) e de Patologia Clínica do Hospital Veterinário (PCHV) da Unidade Acadêmica de Medicina Veterinária (UAMV) do Centro de Saúde e Tecnologia Rural (CSTR) da Universidade Federal de Campina Grande (UFCG), Campus de Patos - PB.

Foram utilizados 30 caprinos da raça moxotó, sendo 15 machos e 15 fêmeas, com idade de 6-12 meses, pesando em média $25 \mathrm{Kg}$, e mantidos em sistema de criação semi- intensivo, em piquetes, recebendo água ad libitum e acesso ao pasto, o qual era composto por extrato herbáceo como as milhãs (Brachiaria plantagínea e B. panicum SP.), capim panasco (Aristida setifolia H.B.K.), capim rabo-deraposa (Setária sp.), com a presença da espécie exótica como o capim buffel (Cenchrus ciliares L.). Entre as dicotiledôneas herbáceas ocorre predominância de matapasto (Senna obtusifolia), malva-branca (Sida cordifolia L), erva-de-orvelha (Stylozanthes sp.), azulão (Centratherum sp.), guizo-de-cascavel (Crotalaria micans Link). Na vegetação lenhosa, destacam-se espécies como a craibeira (Tabebuia aurea), jurema-preta (Mimosa tenuiflora Wild), algaroba (Prosopis juliflora (sw)), juazeiro (Zizyphus joazeiro), bem como as frutíferas, cajarana (Spondias sp.) e cajueiro (Anacardium occidentale).

Os animais foram divididos em cinco grupos: no grupo I, os animais foram tratados com anti-helmíntico à base de moxidectina $0,2 \%{ }^{4}$ em dose única de $0,5 \mathrm{mg} / \mathrm{Kg}$; o grupo II tratado com Typha domingensis Pers na dose (10 $\mathrm{g} / 20 \mathrm{Kg} /$ peso vivo), durante três dias consecutivos; no grupo III, os animais receberam Operculina hamiltonii, na dose de $(09 \mathrm{~g} / 20 \mathrm{Kg} / \mathrm{p} . \mathrm{v}$.$) , durante três dias$ consecutivos; no grupo IV, tratados com $T$. domingensis Pers (10 g/20 Kg/p.v.) associada a O. hamiltonii $(09 \mathrm{~g} / 20$ $\mathrm{Kg} / \mathrm{p} . \mathrm{v}$.), durante três dias consecutivos, todos administrados oralmente e o grupo V (controle) recebeu como tratamento apenas água.

Foram utilizados o rizoma da taboa (Typha domingensis Pers), pertencente à família Thyphaceae e a raiz da batata-de-purga (Operculina hamiltonii (G. Don) D.F. Austin \& Staples), pertencente à família Convolvulaceae, sendo estas coletadas no município de Patos - PB no mês de fevereiro 2007. A exsicata das espécies foi depositada no Herbário Caririense Dárdano de Andrade Lima do Departamento de Biologia da Universidade Regional do Cariri - URCA tendo como registros n ${ }^{\circ} 4021$ e $\mathrm{n}^{\circ} 4022$, respectivamente. Foi realizada a fitoquímica das plantas no laboratório de pesquisa de produtos naturais da Universidade Regional do Cariri, para identificação de seus constituintes químicos, segundo (Matos, 1997).

Amostras individuais de fezes foram coletadas no inicio (dia 0) e aos 07 e 25 dias, diretamente da ampola retal em sacos plásticos devidamente lubrificados com glicerina, identificados e acondicionados em caixas de isopor com gelo até encaminhamento ao LDPAD/CSTR para o processamento dos exames parasitológicos de fezes de acordo com a técnica descrita por Gordon \& Whitlock

${ }^{4}$ Cydectin oral - Fort Dodge ${ }^{\circledR}$ 
(1939), para determinação do número de ovos por grama de fezes (OPG) dos parasitas da família Trichostrongylidae.

$\mathrm{O}$ rizoma e a raiz foram previamente lavados com água esterilizada e, posteriormente, colocados à sombra por um período de $24 \mathrm{~h}$, para secar. Passado esse período, forma levados à estufa de ventilação forçada com temperatura ajustada para $65^{\circ} \mathrm{C}$ por $48 \mathrm{~h}$. Em seguida, realizou-se a moagem em moinho industrial para obtenção do pó, e este foi adicionado à água, o qual foi fornecido por via oral aos animais, segundo adaptação da técnica descrita por Almeida et al. (2007).

Para realização dos exames hematológicos, foi colhido sangue por meio da punção da veia jugular, utilizando-se agulhas descartáveis 40 x $12 \mathrm{~mm}$, após prévia desinfecção do local com álcool iodado. O sangue foi depositado diretamente em frasco de vidro tipo vacutainer, contendo $0,05 \mathrm{~mL}$ de uma solução aquosa a $10 \%$ de etilenodiamino-tetracetato de sódio (EDTA) para cada cinco mL de sangue colhido. As amostras foram mantidas em caixas de isopor com gelo até o encaminhamento ao LPCV/CSTR/ UFCG para o processamento dos exames. As contagens de eritrócitos $(\mathrm{Er})$ foram realizadas em câmara do tipo Neubauer modificada e, para determinação do hematócrito (Ht), utilizou-se a técnica do microhematócrito.

Para determinação do teor de hemoglobina $(\mathrm{Hb})$ no sangue, foi realizada como descrita por Jain (1993) pelo método da cianometahemoglobina, utilizando o analisador bioquímico automático (BIOPLUS 2000), com auxílio de Kit comercial Labtest ${ }^{5}$, próprio para dosagem de hemoglobina e também foram analisados os índices hematimétricos absolutos: volume globular médio (VGM) e concentração de hemoglobina globular média (CHGM), sendo esses exames realizados segundo técnicas de rotina descritas por Coles (1984).

${ }^{5}$ Labtest $^{\circledR}$ - Diagnóstica S.A. Lagoa Santa, MG
No delineamento experimental, os animais foram distribuídos inteiramente ao acaso (DIC), com cinco tratamentos (grupos: I, II, III, IV e V), com seis animais por grupo (seis repetições). Os resultados do OPG foram transformados pela fórmula $\log _{10}(\mathrm{OPG}+1)$ e para análise estatística, os resultados foram submetidos à análise de variância e teste de Tukey a 5\% de significância (SAS Institute, 2003).

\section{RESULTADOS E DISCUSSÃO}

As análises fitoquímicas foram realizadas com o rizoma da taboa (Typha domingensis Pers), que demonstraram presença dos constituintes químicos: catequinas, flavanonas, flavonas, flavononóis, xantonas e taninos flobabênicos (condensados); e a raiz da batatade-purga (Operculina hamiltonii) com presença de: alcalóides, flavanonas, flavonas, flavononóis, xantonas, leucoantocianidinas e taninos flobabênicos (condensados).

$\mathrm{Na}$ contagem do número de ovos por gramas de fezes (OPG) dos grupos tratados, todos apresentaram redução no número médio do OPG aos sete e 25 dias póstratamento, quando comparado com o grupo controle do respectivo período, sendo o grupo da batata-de-purga de maior redução aos sete dias, diferindo estatisticamente apenas do controle, e o grupo da taboa de maior redução aos 25 dias, diferindo dos demais (Tabela 1).

Os taninos presentes em ambas as plantas deste estudo possuem a habilidade de formar complexos insolúveis em água com proteínas, gelatinas e alcalóides, sendo que, ao precipitar proteínas, os taninos propiciam um efeito antimicrobiano e antifúngico. Dentre as hipóteses sobre os mecanismos da ação antimicrobiana dos taninos, destacam-se a inibição de enzimas, a modificação do metabolismo celular pela atuação nas membranas e a complexação com íons metálicos com consequente diminuição da sua disponibilidade para o

Tabela 1 - Número médio de OPGs de caprinos naturalmente infectados em clima semi-árido, submetidos a diferentes tratamentos, nos dias 0,7 e 25 pós-tratamento.

\begin{tabular}{lccc}
\hline \multirow{2}{*}{ Tratamentos } & \multicolumn{3}{c}{ Dias Pós-tratamentos } \\
\cline { 2 - 4 } & $2200( \pm 350) \mathrm{Aa}$ & 7 & \multicolumn{1}{c}{25} \\
\hline Batata-de-purga & $1580( \pm 290) \mathrm{Ba}$ & $700( \pm 350) \mathrm{Bc}$ & $1180( \pm 350) \mathrm{Cb}$ \\
Taboa & $1700( \pm 300) \mathrm{Ba}$ & $538( \pm 500) \mathrm{Bc}$ & $1650( \pm 400) \mathrm{Db}$ \\
Batata-de-purga + Taboa & $1760( \pm 420) \mathrm{Bb}$ & $500( \pm 400) \mathrm{Bc}$ & $2550( \pm 250) \mathrm{Ba}$ \\
Químico (moxidectina) & $1875( \pm 500) \mathrm{Bc}$ & $2300( \pm 550) \mathrm{Ab}$ & $3300( \pm 600) \mathrm{Aa}$ \\
Controle &
\end{tabular}

Letras minúsculas diferentes nas linhas e maiúsculas nas colunas, diferem significativamente $(\mathrm{P}<0,05)$. 
metabolismo dos microorganismos. Entretanto, provavelmente, em razão da habilidade de ligar-se às proteínas e outras macromoléculas, os taninos também apresentam atividades tóxicas, essa toxicidade é decorrente da sua adstringência, e do maior peso da molécula (Mello \& Santos, 2002; Monteiro et al., 2005). Logo, a redução observada na contagem de ovos dos parasitas nas fezes dos animais, está relacionada, possivelmente, com o teor de taninos condensados identificados nas plantas.

De acordo com a metodologia empregada neste trabalho, observou-se que as médias dos valores do VGM, eritrócitos, hematócrito e hemogoblina ficaram dentro dos limites de normalidade, quando comparadas aos valores de referência internacional (Jain, 1993), ficando apenas o CHGM abaixo do limite inferior de normalidade. Segundo diversos autores, os níveis de infestação parasitária por vermes hematófagos digestivos se correlacionam negativamente com parâmetros hematológicos, sendo, as medidas desses índices como o hematócrito e hemoglobina bons indicadores da presença desses parasitos.

Os índices hematimétricos VGM e CHGM indicaram que as células estão normocíticas (normais), e o eritrócito morfologicamente hipocrômico, em razão da redução da concentração de hemoglobina nas hemácias, porém, próximas à normalidades, sendo o grupo controle de menor valor porque estava mais parasitado, uma vez que, não recebeu tratamento, demonstrando, assim, que houve efeito dos tratamentos (Tabela 2).

Para o VGM houve diferença significativa entre os tratamentos $(\mathrm{P}<0,05)$, tendo a associação taboa + batata-de-purga apresentado maior média e o controle a menor, ficando o químico, taboa e batata-de-purga em posição intermediária e não diferindo entre si (Tabela 2).
Isso ocorreu, provavelmente, em decorrência da presença de taninos condensados nas duas plantas, ocasionando uma potencialização, por meio da associação das plantas, proporcionando um maior efeito sobre os parasitos, provocando um declínio na perda de sangue dos animais para os vermes. Esses dados corroboram com Faria Júnior et al. (2002) que obtiveram para caprinos naturalmente infectados com o grupo I com até 500 ovos por grama de fezes (OPG), grupo II (501 a 2000 OPG) e grupo III (mais de 2000 OPG), valores de 18,$32 ; 19,44$ e $20,27 \mathrm{fl}$, respectivamente; Bezerra et al. (2008), avaliando o perfil hematológico de cabras criadas no cariri paraibano, obtiveram média de 19,0 $\mu^{3}$, discordando de Silva et al. (2008) que realizaram avaliação hematológica em caprinos exóticos e nativos no semiárido paraibano, obtendo para raça moxotó VGM 16,97 $\mu^{3}$.

Para o CHGM, não houve diferença significativa entre os tratamentos $(\mathrm{P}>0,05)$, sendo o tratamento com taboa + batata-de-purga de maior média e o controle a menor (Tabela 2). Esses dados foram semelhantes aos de Silva et al. (2008) que obtiveram para a raça moxotó CHGM de 33,60\% e Bezerra et al. (2008) que conseguiram 34,0\%, discordando de Faria Júnior et al. (2002) que obtiveram para os grupos I (+500 OPG) e III (+ 2000 OPG), 38,92 e $35,05 \%$, respectivamente.

No eritrograma (eritrócitos, hematócrito e hemoglobina) a análise de variância revelou efeito dos tratamentos, sendo que, para os Eritrócitos, houve diferença significativa aos sete e 14 dias pós-tratamento, e o grupo controle aos sete dias obteve maior média, diferindo apenas do tratamento com taboa + batata-depurga e sendo igual aos demais (Tabela 3). Esses dados encontrados, provavelmente ocorreram porque, com o passar dos dias e também do efeito dos fitoterápicos,

Tabela 2 - Número médio do Volume Globular Médio (VGM) e Concentração de Hemoglobina Globular Média (CHGM) de caprinos naturalmente infectados por nematóides gastrintestinais em clima semi-árido, submetidos a vários tratamentos.

\begin{tabular}{lcc}
\hline \multirow{1}{*}{ Tratamentos } & VGM & CHGM \\
\cline { 2 - 3 } & $\left(15-30 \mu^{3}\right)^{*}$ & $(35-42 \%)^{*}$ \\
\hline Batata-de-purga + Taboa & $20.19 \mathrm{~A}$ & $32.11 \mathrm{~A}$ \\
Químico (moxidectina) & $19.67 \mathrm{~B}$ & $31.67 \mathrm{~A}$ \\
Taboa & $19.66 \mathrm{~B}$ & $31.49 \mathrm{~A}$ \\
Batata-de-purga & $19.61 \mathrm{~B}$ & $31.44 \mathrm{~A}$ \\
Controle & $18.52 \mathrm{C}$ & $30.33 \mathrm{~A}$ \\
\hline CV $(\%)$ & 2.56 & 6.96 \\
\hline
\end{tabular}

Médias com letras iguais não são significativamente diferentes ( $\mathrm{P}>0,05)$.

*Valores de referência para caprinos (Jain, 1993). 
os animais iam se reinfestando, tendo aumento do parasitismo e consequente alteração no eritrograma; como encontrado por Cenci et al. (2007), que utilizaram Acacia mearnsii em ovinos infectados por helmintos gastrointestinais, onde a concentração do hematócrito e hemoglobina mostrou uma diminuição lenta até o final do experimento, estando perto do limite inferior de $24 \%$ para o hematócrito e $8 \mathrm{~g} / 100 \mathrm{ml}$ para hemoglobina, indicando progressiva perda de sangue, em ambos os tratamentos, mas a perda foi maior no grupo trado sem tanino.

Esses números corroboram com Bezerra et al. (2008) que, em cabras criadas no cariri paraibano, obtiveram média de $14.480,00 \mathrm{~mm}^{3}$, discordando de Faria Júnior et al. (2002) que obtiveram para caprinos naturalmente infectados com os grupos II $(+501$ a 2000 OPG) e III (+ 2000 OPG), valores de eritrócitos de 12.240,00 e 9.320,00, respectivamente.

Para o Hematócrito, aos sete dias, o tratamento de maior média foi da batata-de-purga, diferindo do controle e do grupo da taboa + batata-de-purga, sendo igual aos demais; aos 14 e 28 dias pós-tratamento não houve diferença significativa entre tratamentos e períodos de coletas (Tabela 4). Isso ocorreu, possivelmente, porque a batata-de-purga apresentava mais compostos químicos com efeito parasiticida, como taninos e alcalóides, assim a concentração de hemácias foi maior. Por outro lado, a presença de alcalóides apenas na batata-de-purga talvez tenha provocado um efeito inibidor de outros constituintes químicos presentes na taboa, já que, o grupo tratado com a associação das duas plantas apresentou valores mais baixos para eritrócitos, hematócrito e hemoglobina, aos sete e 14 dias póstratamento.

Esses dados são semelhantes aos de Bezerra et al. (2008) que, em cabras criadas no cariri paraibano, obtiveram média de 27,0\%, discordando de Faria Júnior et al. (2002) para caprinos naturalmente infectados com até 500 OPG (grupo I), obtendo 23.77\%; Silva et al. (2008) obtiveram para hematócrito em caprinos da raça moxotó no, semiárido paraibano, $30.20 \%$.

Em relação à hemoglobina, no período de sete dias pós-tratamento o grupo tratado com batata-de-purga apresentou maior média, diferindo do controle e da associação taboa + batata-de-purga, sendo igual aos demais; aos 14 e 28 dias pós-tratamento não houve diferença significativa entre tratamentos e nem interação com os períodos de coletas (Tabela 5).

$\mathrm{Na}$ ausência dos fatores apropriados a eritropoiese, como por exemplo, os fatores nutricionais, esse processo pode ocorrer de forma anormal, sendo lançadas na circulação eritrócitos com teor de hemoglobina incompleto ou células atípicas, deficientes em número ou com anormalidades fisiológicas (Val-Bicalho \& Carneiro, 2008). Logo, isso poderia ter ocorrido nesse experimento, em razão dos efeitos antinutricionais dos constituintes químicos presentes nas plantas fornecidas aos animais, como sugere Chung et al. (1998), que os taninos parecem ter duplo efeito, por um lado, beneficiam a saúde, em razão de seu efeito e atividades antimicrobianas, por outro lado, estão envolvidos possivelmente na hepatotoxicidade ou efeitos antinutricionais, indicando que, na dosagem e frequência de administração deste estudo esses efeitos não se manifestaram.

Tabela 3 - Número médio de Eritrócitos (Er) em relação aos tratamentos e ao período de coletas (7, 14 e 28 dias póstratamento) de caprinos naturalmente infectados por nematóides gastrintestinais em clima semi-árido, submetidos a vários tratamentos.

\begin{tabular}{lccc}
\hline \multirow{2}{*}{ Tratamentos } & \multicolumn{3}{c}{ Eritrócitos $\left(8-18 \times 10^{6} \mathrm{~mm}^{3}\right)^{*}$} \\
\cline { 2 - 4 } & \multicolumn{3}{c}{ Intervalos das Coletas (dias) } \\
\cline { 2 - 4 } & $14.635,00 \mathrm{Aa}$ & 14 & 28 \\
\hline Batata-de-purga & $15.129,00 \mathrm{Aa}$ & $14.929,00 \mathrm{Aa}$ \\
Controle & $14.129,00 \mathrm{Aa}$ & $14.704,00 \mathrm{Aa}$ & $14.552,00 \mathrm{Aa}$ \\
Químico (moxidectina) & $14.145,00 \mathrm{Aa}$ & $14.032,00 \mathrm{Aa}$ & $14.956,00 \mathrm{Aa}$ \\
Taboa & $13.020,00 \mathrm{Ab}$ & $13.585,00 \mathrm{Ab}$ & $13.856,00 \mathrm{Aa}$ \\
Taboa + Batata-de-purga & & 3.58 & \\
\hline CV $(\%)$ & & & 13.95 \\
\hline
\end{tabular}

Médias com letras iguais maiúsculas na linha e minúsculas na coluna não são significativamente diferentes $(\mathrm{P}>0,05)$.

*Valores de referência para caprinos (Jain, 1993). 
Tabela 4 - Número médio do Hematócrito (Ht) em relação aos tratamentos e ao período de coletas (7, 14 e 28 dias póstratamento) de caprinos naturalmente infectados por nematóides gastrintestinais em clima semi-árido, submetidos a vários tratamentos.

\begin{tabular}{lccc}
\hline \multirow{2}{*}{ Tratamentos } & \multicolumn{3}{c}{ Hematócrito $(19-38 \%)^{*}$} \\
\cline { 2 - 4 } & \multicolumn{3}{c}{ Intervalos das Coletas (dias) } \\
\cline { 2 - 4 } & $28.48 \mathrm{Aa}$ & $28.27 \mathrm{Aa}$ & $27.65 \mathrm{Aa}$ \\
\hline Batata-de-purga & $27.18 \mathrm{Abc}$ & $27.63 \mathrm{Aa}$ & $27.25 \mathrm{Aa}$ \\
Controle & $27.70 \mathrm{Aabc}$ & $27.78 \mathrm{Aa}$ & $27.43 \mathrm{Aa}$ \\
Químico (moxidectina) & $27.85 \mathrm{Aab}$ & $27.70 \mathrm{Aa}$ & $27.87 \mathrm{Aa}$ \\
Taboa & $26.69 \mathrm{Ac}$ & $27.30 \mathrm{Aa}$ & $27.67 \mathrm{Aa}$ \\
Taboa + Batata-de-purga & & 1.99 & \\
CV $(\%)$ & & & \\
\hline
\end{tabular}

Médias com letras iguais maiúsculas na linha e minúsculas na coluna não são significativamente diferentes P> 0,05.

*Valores de referência para caprinos (Jain, 1993).

Tabela 5 - Número médio da Hemoglobina $(\mathrm{Hb})$ em relação aos tratamentos e ao período de coletas (7, 14 e 28 dias póstratamento) de caprinos naturalmente infectados por nematóides gastrintestinais em clima semi-árido, submetidos a vários tratamentos.

\begin{tabular}{lccc}
\hline \multirow{2}{*}{ Tratamentos } & \multicolumn{3}{c}{ Hemoglobina $(8-14 \mathrm{~g} / \mathrm{dl}) *$} \\
\cline { 2 - 4 } & \multicolumn{3}{c}{ Intervalos das Coletas (dias) } \\
\cline { 2 - 4 } & $9.48 \mathrm{Aa}$ & 14 & $9.20 \mathrm{Aa}$ \\
\hline Batata-de-purga & $9.10 \mathrm{Abc}$ & $9.40 \mathrm{Aa}$ & $9.10 \mathrm{Aa}$ \\
Controle & $9.20 \mathrm{Aab}$ & $9.20 \mathrm{Aa}$ & $9.13 \mathrm{Aa}$ \\
Químico (moxidectina) & $9.27 \mathrm{Aab}$ & $9.25 \mathrm{Aa}$ & $9.27 \mathrm{Aa}$ \\
Taboa & $8.90 \mathrm{Ac}$ & $9.23 \mathrm{Aa}$ & $9.20 \mathrm{Aa}$ \\
Taboa + Batata-de-purga & & $9.10 \mathrm{Aa}$ & 1.83 \\
CV $(\%)$ & & $9.05)$. \\
\hline
\end{tabular}

Médias com letras iguais maiúsculas na linha e minúsculas na coluna não são significativamente diferentes $(\mathrm{P}>0,05)$.

*Valores de referência para caprinos (Jain, 1993).

Esses dados corroboram com Faria Júnior et al. (2002) que, para estimar a condição clínica de caprinos parasitados, obtiveram dosagem de hemoglobina de 9,25 g/dl para o grupo I com até 500 OPG, discordando de Cenci et al. (2007), avaliando os efeitos de taninos condensados em ovinos infectados naturalmente com helmintos gastrintestinais, obtendo em sete e 21 dias pós-tratamento, valores de 12.2 e $12.0 \mathrm{~g} / 100 \mathrm{ml}$, respectivamente.

\section{CONCLUSÕES}

De acordo com a metodologia empregada neste experimento a utilização das plantas Typha domingensis
Pers (taboa) e Operculina hamiltonii (batata-de-purga) não interferiu nos valores do VGM, CHGM, eritrócitos, hematócrito e hemoglobina, não alterando a resposta hematológica dos animais.

As plantas utilizadas neste experimento apresentaram potencialidades para programas alternativos de controle de nematóides gastrintestinais de caprinos, sendo, portanto, necessários estudos posteriores para determinação da toxicidade.

\section{AGRADECIMENTOS}

A UFCG e a CAPES pelo auxilio no desenvolvimento desta pesquisa. 


\section{REFERÊNCIAS BIBLIOGRÁFICAS}

ALMEIDA, W.V.F.; SILVA, M.L.C.R.; EDUARDO, B.F.; ATHAYDE, A.C.R.; SILVA, W.W. Avaliação de plantas medicinais em caprinos da região do semi-árido paraibano naturalmente infectados por nematóides gastrintestinais. Revista Caatinga, Mossoró, v.20, n.3, p.1-7, jul./set. 2007.

ATHANASIADOU, S.; KYRIAZAKIS, I. Plant secondary metabolites: antiparasitic effects and their role in ruminant production systems. Proceedings of the Nutrition Society, New York, v.63, p.631-639, 2004.

BEZERRA, L.R.; FERREIRA, A.F.; CAMBOIM, E.K.A.; JUSTINIANO, S.V.; MACHADO, P.C.R.; GOMES, B.B. Perfil hematológico de cabras clinicamente sadias criadas no cariri paraibano. Ciência e Agrotecnologia, Lavras, v.32, n.3, p.955-960, maio/jun. 2008.

CENCI, F.B.; LOUVANDINI, H.; MCMANUS, C.M.; DELL'PORTO, A.; COSTA, D.M.; ARAÚJO, S.C.; MINHO, A.P.; ABDALLA, A.L. Effects of condensed tannin from Acacia mearnsii on sheep infected naturally with gastrointestinal helminthes. Veterinary

Parasitology, London, v.144, p.132-137, 2007.

CHUNG, K.; WEI, C.; JOHNSON, M.G. Are tannins a double-edged sword in biology and healt. Trends in Food Science and Technology, Amsterdam, v.9, n.4, p.168-175, 1998.

COLES, E.H. Patologia clínica veterinária. 3.ed. São Paulo: Manole, 1984. 566p.

DAWSON, J.M.; BUTTERY, P.J.; JENKINS, D.; WOOD, C.D.; GILL, M. Effects of dietary quebracho tannin on nutrient utilisation and tissue metabolism in sheep and rats. Journal of the Science of Food and Agriculture, Bethesda, v.79, p.1423-1430, 1999.

FARIA JÚNIOR, S.P.; SILVA, M.M.; SCHEIBEL, M.; MARTINS, M.F.; RABELLO, P.; BERTAGNON, H.G.; GARCIA, M. Uso da contagem fecal de ovos de nematóides (OPG) para estimar a condição clínica em caprinos. Ciências Veterinárias nos Trópicos, Recife, v.5, n.2/3, p.86-92, 2002.

GORDON, H.H.; WHITLOCK, H.V. A new technique for couting nematode eggs in sheep faeces. Journal
Council Scientific Industry Research, London, v.12, p.50-52, 1939.

HARBORNE, J.B. An overview of antinutritional factors in higher plants. In: CAYGILL, J.C.; MUELLER-HARVEY, I. (Eds.). Secondary plant products: antinutritional and beneficial actions in animal feeding. Nottingham: Nottingham University, 1999. p.7-16.

JAIN, N.C. Essentials of veterinary hematology.

Philadelphia: [s.n.], 1993. 417p.

MATOS, F.J.A. Introdução à fitoquímica experimental. Campina Grande: UFCG, 1997. 46p.

MELLO, C.P.; SANTOS, S.C. Taninos. In: SIMÕES, C.M.O. (Org.). Farmacognosia: da planta ao medicamento. 4.ed. Porto Alegre: UFRGS; Florianópolis: UFSC, 2002.

MIN, B.R.; BARRY, T.N.; ATTWOOD, G.T.; MCNABB, W.C. The effect of condensed tannins on the nutrition and health of ruminants fed fresh temperate forages: a review. Animal Feed Science and Technology, Oxford, v.106, p.3-19, 2003.

MONTEIRO, J.M.; ALBUQUERQUE, U.P.; ARAÚJO, E.L.; AMORIM, E.L.C. Taninos: uma abordagem da química à ecologia. Química Nova, São Paulo, v.28, n.5, p.892-896, set./out. 2005.

SAS INSTITUTE. SAS user's guide: statistics. Version 8.2. Cary, 2003.

SILVA, E.M.N.; SOUZA, B.B.; SILVA, G.A.; CÉZAR, M.F.; FREITAS, M.M.S.; BENÍCIO, T.M.A. Avaliação hematológica de caprinos exóticos e nativos no semiárido paraibano. Ciência e Agrotecnologia, Lavras, v.32, n.2, p.561-566, mar./abr. 2008.

VAL-BICALHO, A.P.C.; CARNEIRO, R.A. Patologia clínica. Belo Horizonte: UFMG, 2008. 85p. Apostila. Disponível em: <http://Www.vet.ufmg.br/departamentos/1 iclinica/clinica/disciplinas/patologia-clinica-ccv-003 Acesso em: 30 set. 2008.

WAGHORN, G.C.; MCNABB, W.C. Consequences of plant phenolic compounds for productivity and health of ruminants. Proceedings of the Nutrition Society, New York, v.62, p.383-392, 2003. 